\title{
Absortâncias e emitâncias efetivas de superfícies: um método para estimá- las, um modelo experimental e exemplos de suas importâncias
}

\author{
Effective surface absorbtances and emittances: an \\ estimating method, an experimental model, and
} examples of their importance

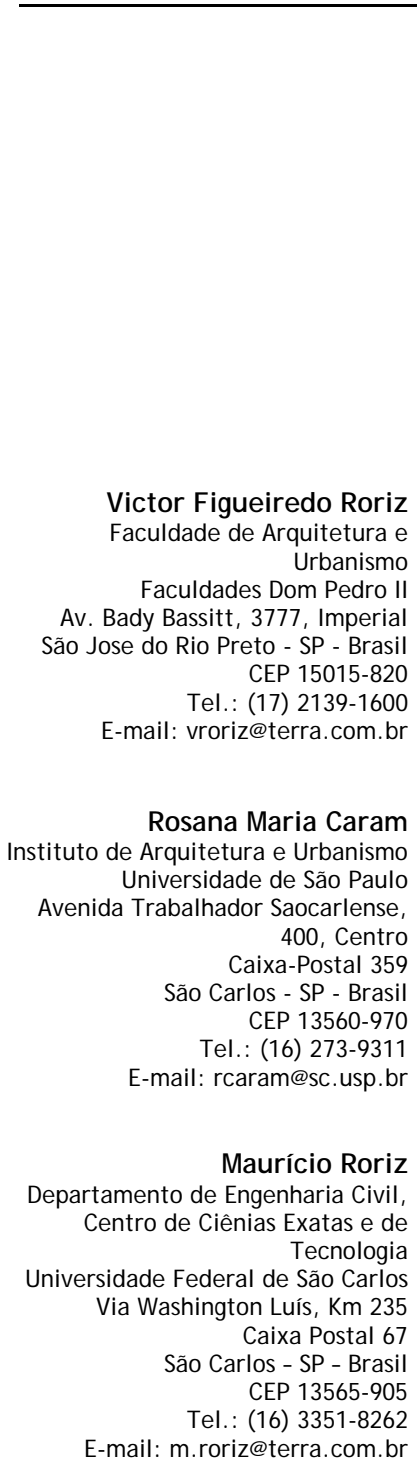

Recebido em 14/02/12

Aceito em 21/05/12

\section{Victor Figueiredo Roriz \\ Rosana Maria Caram Maurício Roriz}

\section{Resumo}

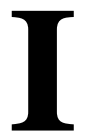

mperfeições, saliências e reentrâncias existentes nas superfícies típicas das edificações constituem obstáculos que podem alterar significativamente o comportamento delas em relação aos fluxos por radiação. Além de ampliar a área efetiva de troca de calor, criam sombras e reflexões, que não ocorreriam em. Assim, os procedimentos de cálculo, ao invés de considerar as superfícies perfeitamente lisas e planas, deveriam aplicar correções nas propriedades radiativas das superfícies. Denominam-se efetivas as absortâncias e emitâncias que resultam dessas correções. O presente artigo apresenta um método simples desenvolvido para avaliar essas influências e exemplifica a importância das correções acima mencionadas, apresentando resultados de simulações elaboradas no programa EnergyPlus para uma edificação de geometria simples, submetida ao longo de 1 ano ao clima da cidade de Brasília. Adotando-se absortâncias e emitâncias efetivas, os resultados das simulações revelam diferenças de até $2,8^{\circ} \mathrm{C}$ nas temperaturas internas do ar em relação às obtidas desprezando-se as influências que as ondulações das telhas exercem sobre os fluxos radiantes. Considerando o uso de condicionadores de ar, essa diferença pode representar uma variação de cerca de 30\% nas estimativas de consumo de energia.

Palavras-chave: Absortividade. Emissividade. Absortância efetiva. Emitância efetiva.

\section{Abstract}

Building surfaces usually have bumps and hollows that can significantly change their behaviour in relation to radiant flow. Therefore, the usual calculation procedures, instead of adopting the simplification that all surfaces of a construction are perfect plans, should apply corrections in the radiative properties of those surfaces. The effective absorptances and emittances resulting from those surface irregularities should be considered. This paper presents a simple method to evaluate those influences and exemplifies the importance of such aspects, presenting results of simulations developed in EnergyPlus for a simple geometry building, over a one-year period, in the city of Brasilia. Adopting effective absorptances and emittances, simulation results show differences of up to $2.8^{\circ} \mathrm{C}$ in the internal air temperature, compared to those obtained ignoring the influences that the ripples of the tiles have on radiant flows. Considering the use of air conditioners, this could represent up to $30 \%$ variation in the energy consumption estimate.

Keywords: Absorptivity. Emissivity. Effective emittances. Effective absorptances. 


\section{Introdução}

Nos cálculos de fluxo de calor e avaliação de consumo energético, as superfícies dos edifícios são geralmente tratadas como planos perfeitos, sem ondulações ou rugosidade. Entretanto, nesta simplificação, alguns cuidados devem ser tomados a fim de serem consideradas as características reais das superfícies.

Diferentes definições são encontradas na literatura para as propriedades radiativas das superfícies. No presente trabalho, adotam-se os sufixos "-ade”, para propriedades de materiais, e “-ância”, para propriedades de corpos ou superfícies. Dessa forma, as absortividades e as emissividades são propriedades dos materiais, enquanto as absortâncias e as emitâncias são características das superfícies, sendo influenciadas não apenas pelo material de que são constituídas, mas também por sua geometria e acabamento superficial, que podem provocar sombras e inter-reflexões.

Vale ressaltar que os procedimentos usuais de avaliação de absortâncias solares são relacionados apenas às cores das superfícies, sendo estas classificadas em 5 ou 6 intervalos, de modo extremamente impreciso, principalmente por desconsiderarem as parcelas não visíveis da radiação solar, nas faixas de infravermelho e ultravioleta. Para a avaliação da emissividade esse fato é ainda mais impreciso, adotando-se apenas duas ou três categorias para sua classificação. Além disso, muitas vezes se confundem propriedades do material e propriedades da superfície, e suas diferenças são ignoradas ou desprezadas.

Superfícies perfeitamente lisas e planas não oferecem obstáculos à troca radiativa de calor. No entanto, saliências e reentrâncias das superfícies, tanto na escala da rugosidade quanto das ondulações (de uma telha, por exemplo), podem alterar significativamente o comportamento delas em relação aos fluxos por radiação (BERDAHL; BRETZ, 1997; SEKER; TAVIL, 1996).

Na avaliação da emissividade, por exemplo, normas internacionais de medição dessa propriedade, como a ASTM C1371 (AMERICAN..., 2004) e a JIS A 1423 (JAPANESE..., 1983), exigem que as amostras sob análise sejam lisas e planas. Também a ASTM C1549 (AMERICAN..., 2009) faz a mesma exigência para a medição da refletância. Para superfícies perfeitamente planas e lisas, refletividade e refletância equivalem-se.

No tocante à radiação, é possível estimar o efeito que as irregularidades criam e adotar a simplificação de planos perfeitos, através do uso de propriedades efetivas para as superfícies. Nesse caso, o termo "efetivo" aplica-se às absortâncias e emitâncias corrigidas em função das irregularidades superficiais. Sparrow e Jonsson (1963) modelaram esse efeito para algumas superfícies e demonstraram a importância do fenômeno. A dedução apresentada por esses autores, entretanto, aplica-se a geometrias simples e específicas, restringindo sua aplicação a uma série limitada de casos. No presente artigo, apresenta-se um modelo simplificado, válido para qualquer geometria, e comparam-se seus resultados aos obtidos pelo método de referência citado. Apresenta-se, ainda, um experimento que demonstra os efeitos desse fenômeno e que exemplifica sua importância.

Na avaliação dos fluxos de calor e da eficiência energética de um ambiente, esses efeitos podem ser muito relevantes. No caso de avaliações de coberturas, por exemplo, uma telha pode ter seu comportamento térmico diferenciado de outra, dependendo de suas formas, mesmo que sejam feitas do mesmo material e tenham a mesma pintura, por exemplo. No caso de uma parede, a adoção de uma textura pode influenciar o comportamento radiativo e a temperatura da superfície.

O modelo apresentado permite estimar essa influência e, assim, pode contribuir para a elaboração de cálculos mais realistas. Esse modelo foi desenvolvido ao longo de uma pesquisa de doutorado (RORIZ, 2011).

Este artigo está dividido em três partes:

(a) equacionamento dos efeitos geométricos nas propriedades radiativas de uma superfície;

(b) realização de um experimento que ilustre e comprove os fenômenos aqui abordados; e

(c) simulações, no programa EnergyPlus, do comportamento térmico de edificações considerando ou não os efeitos das absortâncias e emitâncias efetivas.

\section{Equacionamento}

Considere-se uma superfície isotérmica e difusa que emita calor e o entorno dela, representados na Figura 1 pelas superfícies 1 e 2 respectivamente. Se toda a energia emitida pela superfície 1 (E) for representada em um único ponto $\left(\mathrm{P}_{0}\right)$, uma parcela dela poderá ser emitida para o exterior (vetor $\vec{A}$ ), e uma segunda parcela (vetor $\vec{B}$ ), em direção à própria superfície (Eq. 1 e 2 respectivamente). 


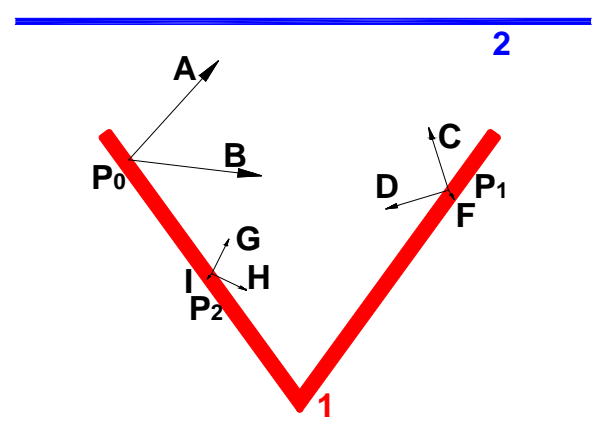

Figura 1 - Energia emitida por uma superfície côncava (1) para seu entorno (2)

\begin{tabular}{|l|l|l|}
\hline Efetivamente emitida para a sup. 2 & Emitida sobre a superfície 1 & Absorvida pela superfície 1 \\
\hline$\vec{A}=E . F F_{12}$ & $\vec{B}=E \cdot F F_{11}$ & - \\
\hline$\vec{C}=E \cdot F F_{11} \cdot \rho \cdot F F_{12}$ & $\vec{D}=E \cdot F F_{11}{ }^{2} \cdot \rho$ & $\vec{F}=E . F F_{11} \cdot \alpha$ \\
\hline$\vec{G}=E \cdot F F_{11}{ }^{2} \cdot \rho^{2} \cdot F F_{12}$ & $\vec{H}=E \cdot F F_{11}{ }^{3} \cdot \rho^{2}$ & $\vec{I}=E . F F_{11}{ }^{2} \cdot \rho \cdot \alpha$ \\
\hline
\end{tabular}

Quadro 1 - Energia emitida pela superfície 1

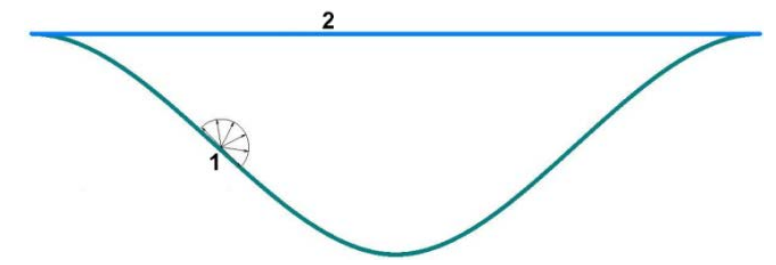

Figura 2 - Corte de uma cavidade

Os seguintes símbolos são adotados para as equações apresentadas nesse trabalho:
A: área $\left(\mathrm{m}^{2}\right)$;
E: energia emitida (J);
FF: fator de forma
$\alpha$ : absortividade - absortância;
$\rho$ : refletividade - refletância; e
$\varepsilon$ : emissividade - emitância.
Para símbolos subscritos:
e: efetivo;

$\mathrm{CN}$ : corpo negro;

1: referente à superfície 1 ;

2: referente à superfície 2; e

a: absorvida.

$$
\begin{aligned}
& \vec{A}=E \cdot F F_{12} \\
& \vec{B}=E \cdot F F_{11} \\
& 2
\end{aligned}
$$

$\mathrm{O}$ fator de forma $\left(\mathrm{FF}_{12}\right)$ da superfície 1 em relação à superfície 2 é a razão entre a parcela de toda a

Eq. 1 energia radiante que sai de 1 e é interceptada por 2 e a energia radiante que sai de 1 . Pelo mesmo conceito, $\mathrm{FF}_{11}$ refere-se à parcela de energia emitida por 1 e que é interceptada pela própria superfície 1.

Considerando que a parcela representada por $\vec{B}$ incida sobre um ponto $\mathrm{P}_{1}$ da superfície 1 , uma fração de $\vec{B}$, que denominaremos $\vec{C}$, também será refletida para o exterior, somando-se ao vetor $\vec{A}$, enquanto uma parte $\vec{D}$ será refletida sobre a própria superfície e uma parcela $\vec{F}$ será absorvida pela superfície. Repetindo o raciocínio, é possível considerar então três opções. Na primeira, a energia efetivamente sai da superfície 1 e alcança a superfície 2. Na segunda, a energia sai de 1 e volta para 1. Na terceira, a energia é absorvida pela superfície 1. O Quadro 1 resume esses casos. Vale destacar que a absortância $(\alpha)$ e a refletância $(\rho)$ referem-se aos intervalos de comprimentos de onda pertinentes a cada estudo.

Eq. A parcela de $\mathrm{E}$ que efetivamente é emitida para 2 $\left(\mathrm{E}_{\mathrm{e}}\right)$ pode, então, ser calculada pela série (Equação 3):

$$
E_{e}=\vec{A}+\vec{C}+\vec{G}+\ldots
$$




$$
E_{e}=E \cdot F F_{12}+E \cdot F F_{11} \cdot \rho_{1} \cdot F F_{12}+E \cdot F F_{11}^{2} \cdot \rho_{1}^{2} \cdot F F_{12}+\ldots \quad \varepsilon=\alpha=1-\rho
$$

Eq. 3 Portanto (Equação 15):

Resolvendo-se a série, obtém-se:

$$
E_{e}=\frac{E \cdot F F_{12}}{1-\left(F F_{11} \cdot \rho_{1}\right)}
$$

Portanto, a energia que realmente sai da cavidade, ou seja, que cruza a superfície 2 (reta na Figura 2), pode ser calculada pela Equação 4.

Portanto, a energia que realmente sai da cavidade, ou seja, que cruza a superfície 2 (reta na Figura 2), pode ser calculada pela Equação 4.

Considerando a superfície de estudo como uma cavidade (como a exemplificada pela superfície 1 na Figura 2) e seu entorno como um plano que limita essa cavidade (superfície 2 na Figura 2), é possível considerar a Equação 5 e calcular pela Equação 6 os fatores de forma entre a superfície e o entorno.

$$
\begin{aligned}
& F F_{11}=1-F F_{12} \\
& F F_{12}=\left(\frac{A_{2}}{A_{1}}\right)
\end{aligned}
$$

De 4 e 5 (Equação 7):

$$
E_{e}=\frac{E \cdot F F_{12}}{1-\rho_{1}+F F_{12} \cdot \rho_{1}}
$$

Da definição de emitância (Equação 8):

$$
\varepsilon=\frac{E}{E_{C N}}
$$

Ond (Equações 9 e 10) e:

$$
\begin{aligned}
& E=A_{1} \cdot \varepsilon_{1} \cdot \sigma \cdot T_{1}^{4} \\
& E_{C N}=A_{2} \cdot \sigma \cdot T_{1}^{4}
\end{aligned}
$$

Aplicando 7 e 10 em 8, vem (Equação 11):

$$
\varepsilon_{e}=\frac{\frac{E \cdot F F_{12}}{1-\rho_{1}+F F_{12} \cdot \rho_{1}}}{A_{2} \cdot \sigma \cdot T_{1}^{4}}
$$

Aplicando 9 em 11 e manipulando a Equação 12, obtém-se:

$\varepsilon_{e}=\frac{\varepsilon_{1} \cdot F F_{12}}{1-\rho_{1}+F F_{12} \cdot \rho_{1}} \cdot \frac{A_{1}}{A_{2}}$

De 12 e 6, vem (Equação 13):

$$
\varepsilon_{e}=\frac{\varepsilon_{1}}{1-\rho_{1}+F F_{12} \cdot \rho_{1}}
$$

Considerando a superfície como cinza, da lei de Kirchhoff, vem (Equação 14):

$$
\varepsilon_{e}=\frac{\varepsilon_{1}}{\varepsilon_{1}+F F_{12}-\varepsilon_{1} F F_{12}}
$$

Ou, de 6 (Equação 16):

$$
\varepsilon_{e}=\frac{\varepsilon_{1} \cdot A_{1}}{A_{2}-\varepsilon_{1} \cdot A_{2}+\varepsilon_{1} \cdot A_{1}}
$$

Um raciocínio similar pode ser desenvolvido para a absortância. Através da soma de $n$ parcelas absorvidas, fruto de n-1 inter-reflexões, obtém-se a seguinte Equação (17) para a energia absorvida $\left(E_{a}\right)$ considerando uma irradiância I:

$$
\begin{array}{ll}
E_{a}=\alpha_{e} \cdot I=\alpha_{1} \cdot I+\left(\alpha_{1} \cdot F F_{11} \cdot \rho_{1} \cdot I\right)+\left(\alpha_{1} \cdot F F_{11}^{2} \cdot \rho_{1}^{2} \cdot I\right)+\ldots \\
E_{a}=\alpha_{e} \cdot I=\sum_{i=0}^{n} \alpha_{1} \cdot F F_{11}{ }^{i} \cdot \rho_{1}{ }^{i} \cdot I & \text { Eq. } 17
\end{array}
$$

E, portanto, resolvendo a série, obtém-se (Equação 18):

$$
\alpha_{e}=\frac{\alpha_{1}}{1-\left(F F_{11} \cdot \rho_{1}\right)}
$$

Para uma superfície opaca $(\alpha+\rho=1)$, fica (Equação 19):

$$
\alpha_{e}=\frac{\alpha_{1}}{1-F F_{11}+F F_{11} \cdot \alpha_{1}}
$$

Ou, considerando as equações 5 e 6 (Equação 20):

$$
\alpha_{e}=\frac{\alpha_{1} \cdot A_{1}}{A_{2}-\alpha_{1} \cdot A_{2}+\alpha_{1} \cdot A_{1}}
$$

Assim, através das Equações 16 e 20, é possível calcular os valores efetivos das emitâncias e absortâncias respectivamente, e os mesmos podem apresentar diferenças significativas em relação aos valores de referência.

Na modelagem da Equação 19 não há qualquer restrição ao comprimento de onda; portanto, esse desenvolvimento vale também para as frequências da radiação solar, onde grande impacto pode ser esperado pela alta quantidade de energia envolvida.

O efeito da forma na emitância efetiva, calculado pela Equação 16, é apresentado na Figura 3. Nessa figura, é possível observar que a redução da razão entre as áreas $A_{2}$ e $A_{1}$, indicada pela redução de $\mathrm{FF}_{12}$ (vide Equação 6), implica o aumento da emitância efetiva da superfície teórica (superfície 2 na Figura 2). Na realidade, esse efeito decorre da maior área de emissão de calor.

Sparrow e Jonsson (1963) desenvolveram modelos bastante precisos para a avaliação desse efeito. 
Entretanto, seu método de cálculo é baseado em integrações para as superfícies de estudo, sendo, assim, aplicável a casos bastante restritos e específicos.

Comparando os resultados obtidos pela Equação 16 aos apresentados por Sparrow e Cess (1978), obtêm-se as curvas apresentadas nas Figuras 4 e 5 Na Figura 4, o eixo das abscissas representa a razão entre a profundidade e a espessura da cavidade (L/h). Na Figura 5 esse eixo representa o ângulo de abertura da cavidade $(\phi)$.

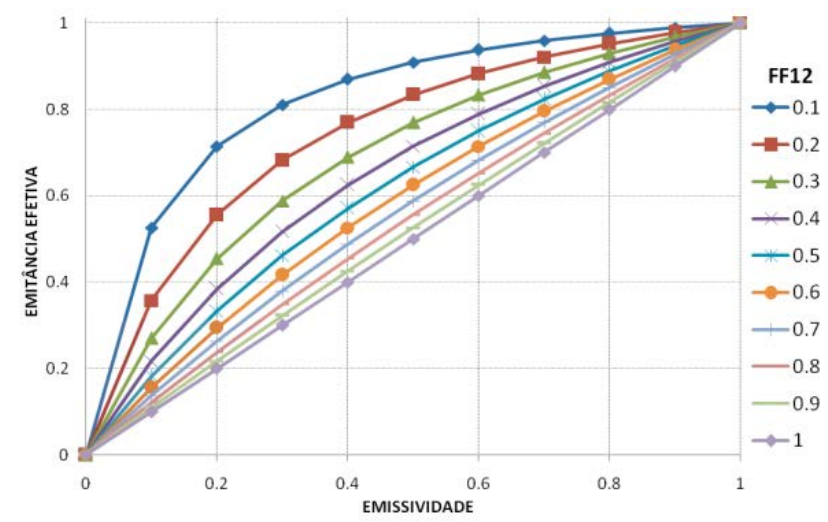

Figura 3 - Efeito da forma na emitância efetiva (Eq. 15)

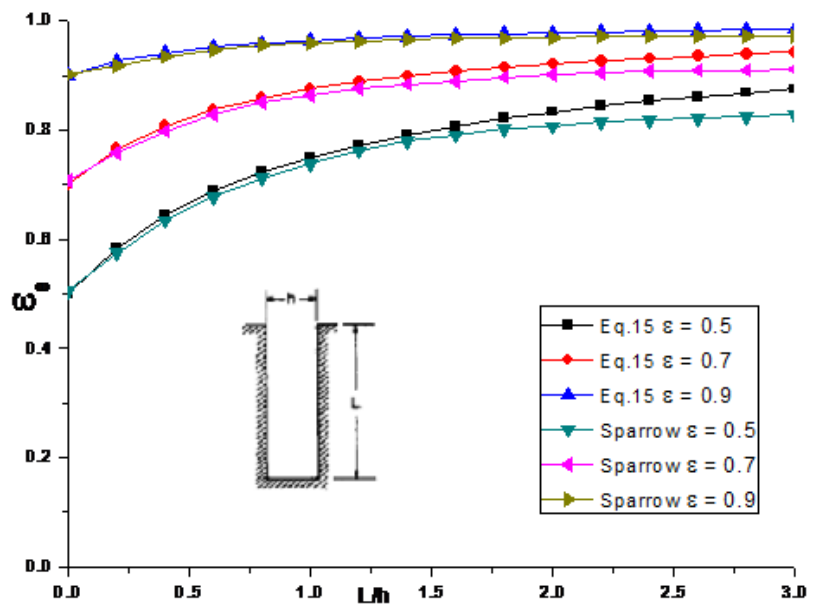

Figura 4 - Efeito cavidade para ranhura retangular

Nota: comparação da Equação 16 a resultados teóricos apresentados por Sparrow e Cess (1978).

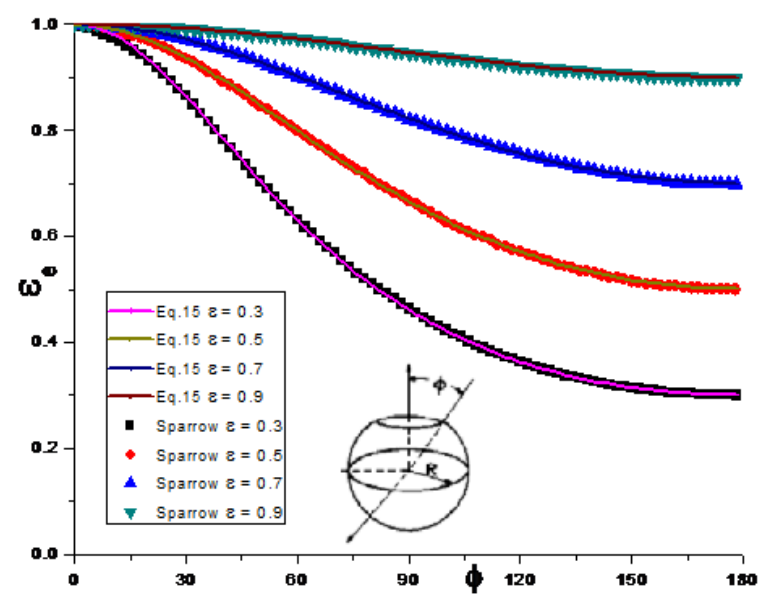

Figura 5 - Efeito cavidade para cavidade esférica

Nota: comparação da Equação 16 a resultados teóricos apresentados por Sparrow e Cess (1978). 
A diferença entre esses modelos é resultado de que, no modelo proposto por Sparrow e Jonsson, considera-se a variação na radiosidade entre os pontos da superfície. No caso da ranhura retangular (Figura 4), por exemplo, a proximidade da abertura implicaria radiosidades mais altas que em pontos mais profundos, implicando também um limite a esse efeito na cavidade. No modelo aqui proposto, considera-se que todos os pontos da superfície tenham o mesmo comportamento. Para cavidades esféricas, as soluções são idênticas, enquanto para ranhuras retangulares a aproximação é admissível até uma relação $\mathrm{L} / \mathrm{h}$ de aproximadamente 1,5, conforme indicam as Figuras 4 e 5 . Esses limites satisfazem à maior parte dos componentes da construção civil.

As vantagens do uso da Equação 16 são sua simplicidade de cálculo e sua possibilidade de aplicação a quaisquer formas.

\section{Experimento}

A Figura 6 apresenta um corte esquemático do experimento. Em uma câmara de vácuo e por meio de uma bomba de vácuo, criou-se uma atmosfera rarefeita, a fim de minimizar os possíveis fluxos de calor por convecção. Além disso, as amostras tiveram seus fundos e laterais recobertos por isolante, minimizando outros fluxos de calor e permitindo registrar os efeitos provocados apenas pelas trocas térmicas por radiação em ondas longas entre as amostras e a câmara.

Como câmara de vácuo, foi adaptada uma panela de pressão que, por ser hermética, opaca, fabricada em alumínio (alta condutividade), com dimensões adequadas e superfícies polidas (baixa emissividade), proporcionou as condições necessárias ao experimento.

$\mathrm{O}$ procedimento experimental consistiu em provocar bruscas alterações na temperatura da câmara de vácuo, registrando ao longo do tempo as alterações sofridas nas temperaturas de cada amostra. Esse objetivo foi alcançado submergindo a câmara em água gelada ou fervente.

Cada amostra foi composta de duas peças (Figura 6), uma feita de papel-alumínio, com $17 \mathrm{~cm} \mathrm{x} 6$ cm, colada a uma folha de papel (apenas para facilitar a montagem, Figura 7). Esse conjunto foi encurvado, de modo a formar um cilindro de 2" de diâmetro (Figura 8). A seção transversal (circular) dessa peça define uma área denominada $\mathrm{A}_{2}$ (Figura 6).

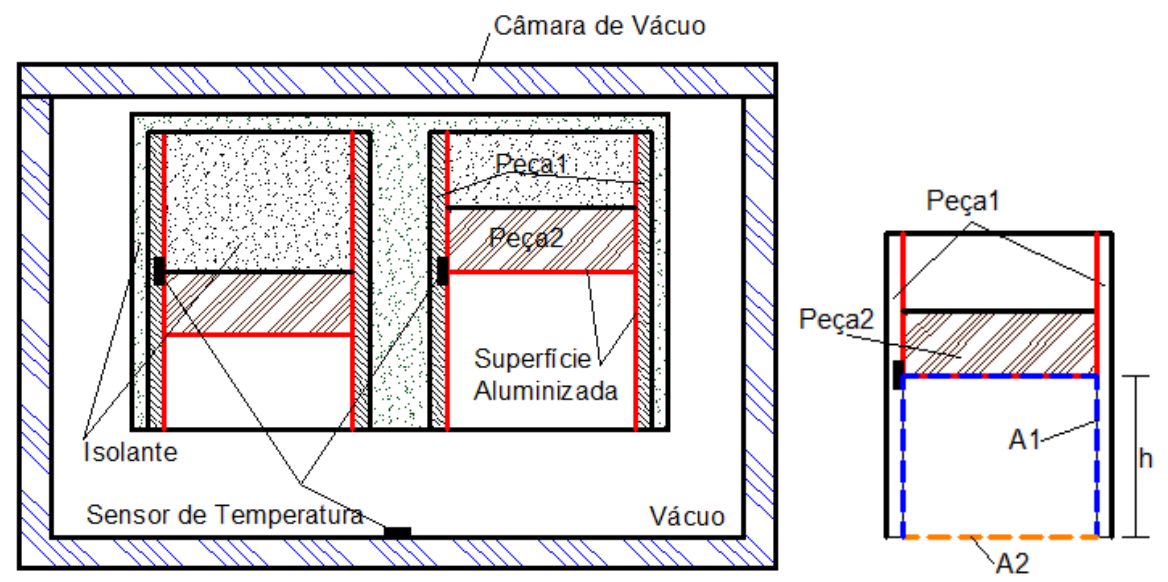

Figura 6 - Corte esquemático do experimento e detalhe de amostra

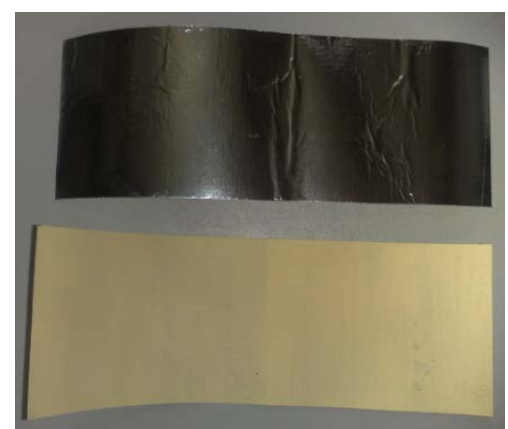

Figura 7 - Montagem das amostras, material para peça 1 


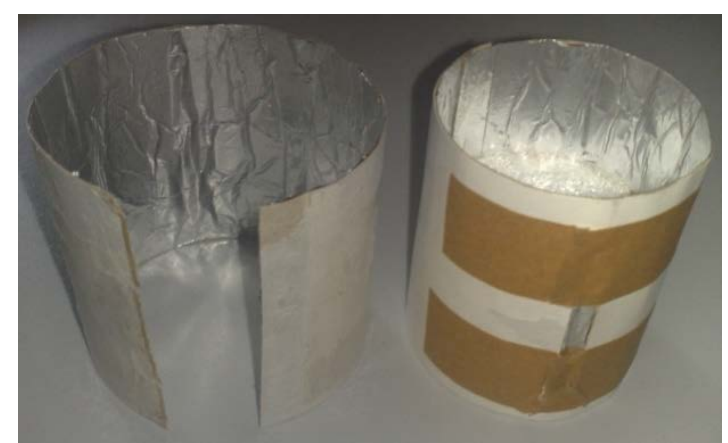

Figura 8 - Montagem das amostras, peça 1

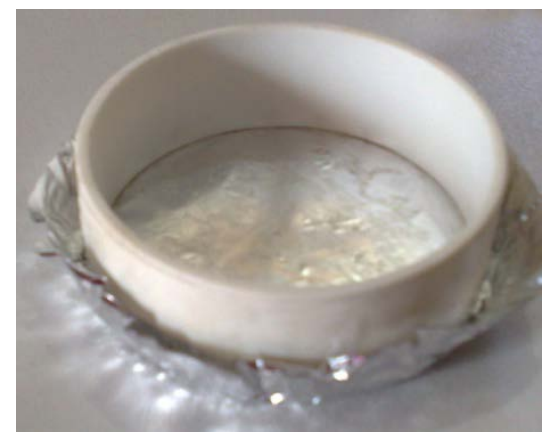

Figura 9 - Montagem das amostras, material para peça 2

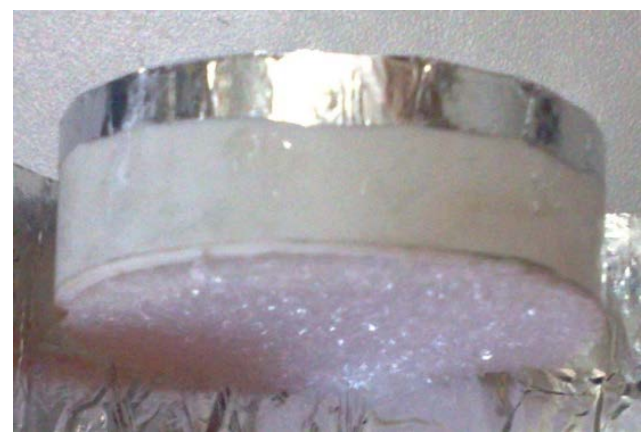

Figura 10 - Montagem das amostras, peça 2

A segunda peça das amostras foi feita dobrando-se e colando-se um disco de papel-alumínio sobre um anel de PVC de 2" de diâmetro e 1,5 cm de altura (Figura 9), e preenchendo esse conjunto com material isolante (Figura 10). Além da fixação, a lateral dobrada teve a função de manter o contato térmico entre as peças. $\mathrm{O}$ anel de PVC deu rigidez ao conjunto, além de ajudar a manter a perpendicularidade entre as peças.

A segunda peça foi fixada no interior da primeira, em diferentes alturas (Figura 11), conforme a Tabela 1. A área de superfície aluminizada exposta à troca de calor, denominada $\mathrm{A} 1$, ficou, portanto, diferenciada para cada amostra. Essa área A1 é a soma da superfície exposta da peça 2 e da parcela exposta da superfície interna da peça 1 (Figura 6).
Considerando como cavidade fechada o espaço entre as áreas $A_{1}$ e $A_{2}$, é possível calcular o fator de forma entre as superfícies pela Equação 6.

Dessa forma, o deslocamento da peça 2 em relação à peça 1 (h na Figura 6) fez com que cada amostra tivesse um fator de forma diferente, mantendo idênticas, entretanto, suas demais características (massa, emissividade, área do corte transversal, etc.).

Na lateral de cada amostra (Figura 11), recortes foram previamente feitos no papel, para possibilitar a fixação de sensores de temperatura diretamente sobre o papel-alumínio (Figura 12). Nesse experimento, utilizaram-se sensores modelo DS18B20 One-wire Thermometer, fabricados pela Maxim, com resolução de 0,0625 ${ }^{\circ} \mathrm{C}$ (MAXIM, 2011). 


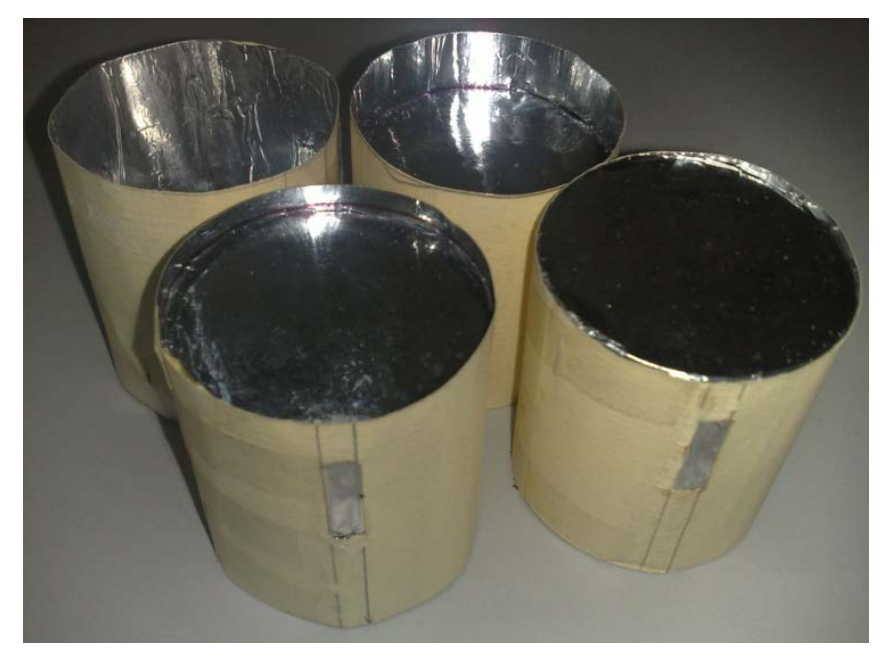

Figura 11 - Amostras com diferentes fatores de forma

Tabela 1 - Fatores de forma

\begin{tabular}{lccc}
\hline \multicolumn{1}{c}{$\mathbf{F F}_{\mathbf{1 2}}$} & $\mathbf{A}_{\mathbf{2}}\left(\mathbf{c m}^{\mathbf{2}}\right)$ & $\mathbf{A}_{\mathbf{1}} \mathbf{( \mathbf { c m } ^ { 2 } )}$ & $\mathbf{h} \mathbf{( c m )}$ \\
\hline 0,25 & 20,268 & 81,0732 & 3,810 \\
0,5 & 20,268 & 40,5366 & 1,270 \\
0,75 & 20,268 & 27,0244 & 0,423 \\
1 & 20,268 & 20,2683 & 0,000 \\
\hline
\end{tabular}

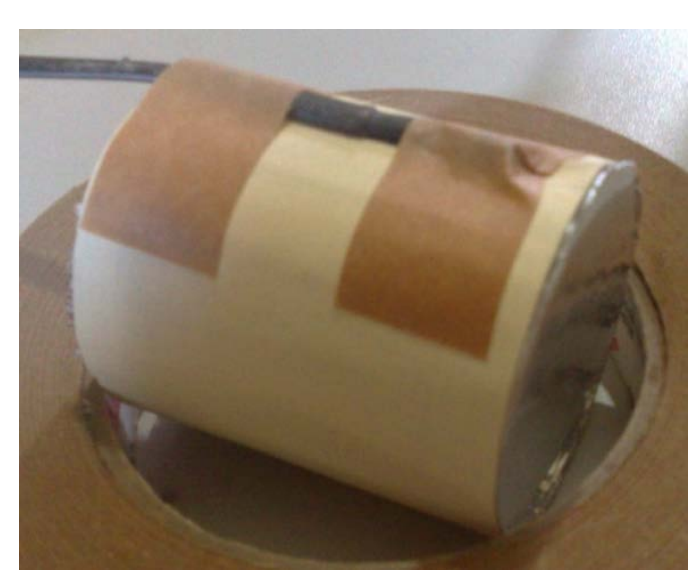

Figura 12 - Fixação dos sensores

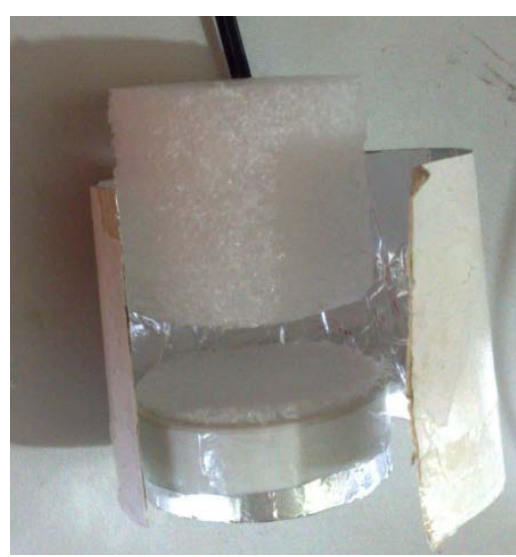

Figura 13 - Preenchimento com isolante

14 Roriz, V. F.; Caram, R. M.; Roriz, M. 
A região do cilindro acima da peça 2 foi preenchida com isolante (Figura 13). Para minimizar fluxos de calor por condução, as amostras foram encaixadas duas a duas em blocos de isolantes (Figuras 14 e 15).

Fixou-se um sensor de temperatura no centro do fundo da câmara e um segundo sensor à meia altura de sua lateral (Figura 16). O conjunto de amostras (Figura 17) foi instalado no interior da câmara, com as superfícies aluminizadas voltadas para baixo (Figura 18). O orifício onde havia uma válvula de segurança foi utilizado como passagem do cabo dos sensores. A câmara foi fechada e lacrada com silicone (Figura 19).

Para controlar a realização do experimento, uma válvula e um indicador de vácuo foram adaptados à conexão com a bomba de vácuo (Figura 20).

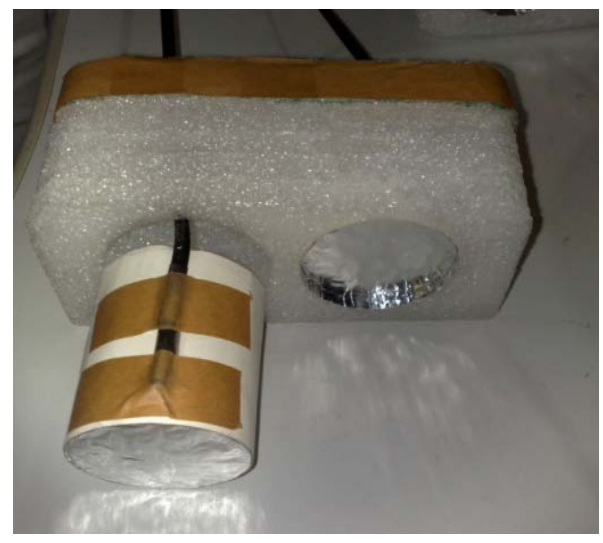

Figura 14 - Montagem das amostras

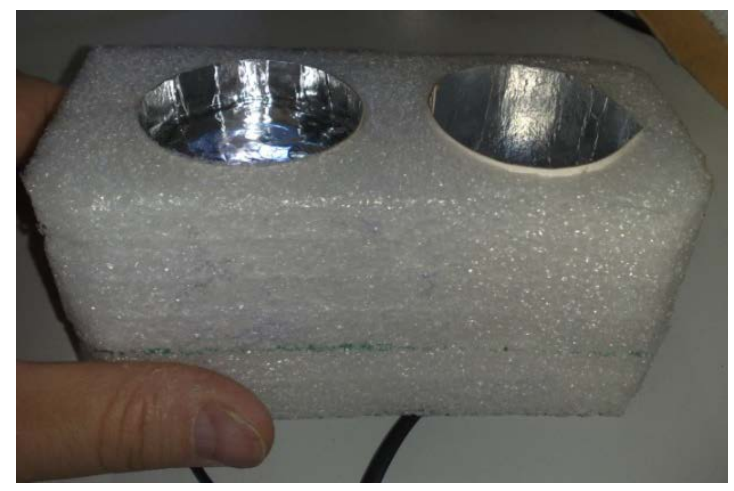

Figura 15 - Montagem das amostras no bloco isolante

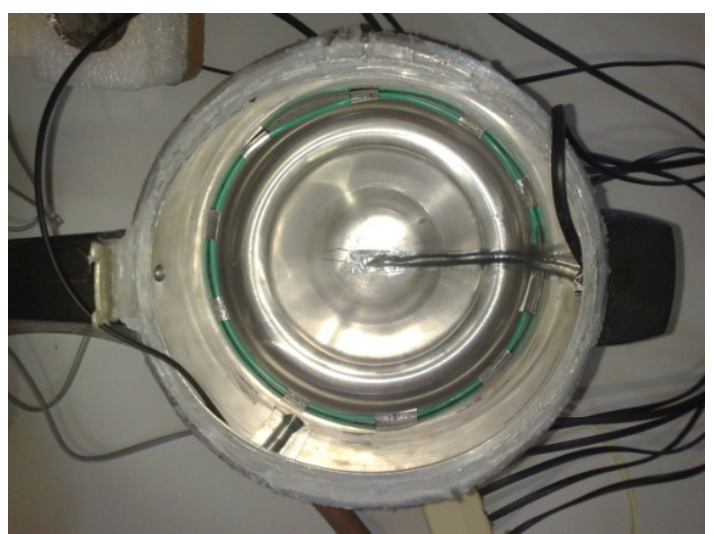

Figura 16 - Vista superior da câmara de vácuo 


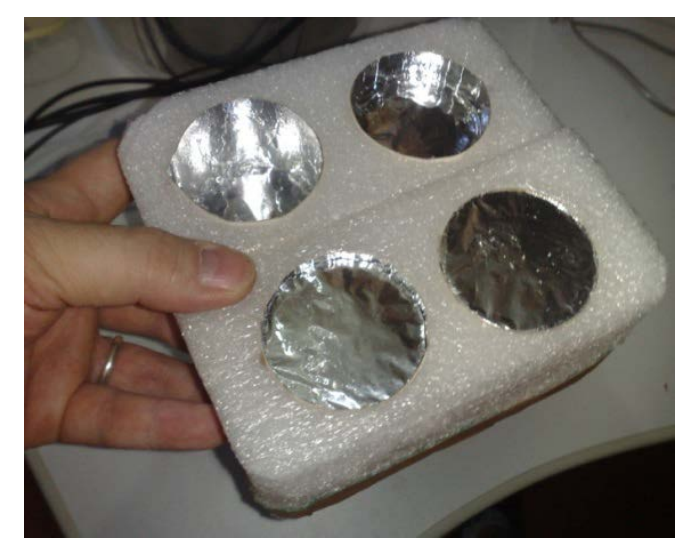

Figura 17 - Conjunto das amostras (vista inferior)

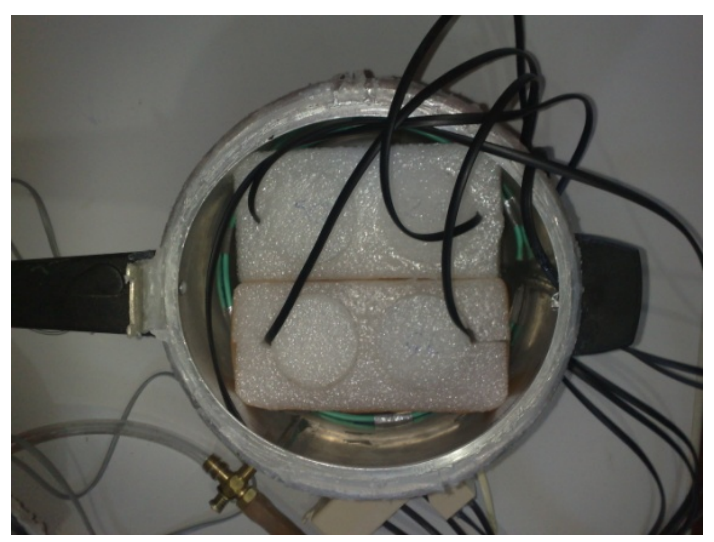

Figura 18 - Câmara de vácuo com as amostras

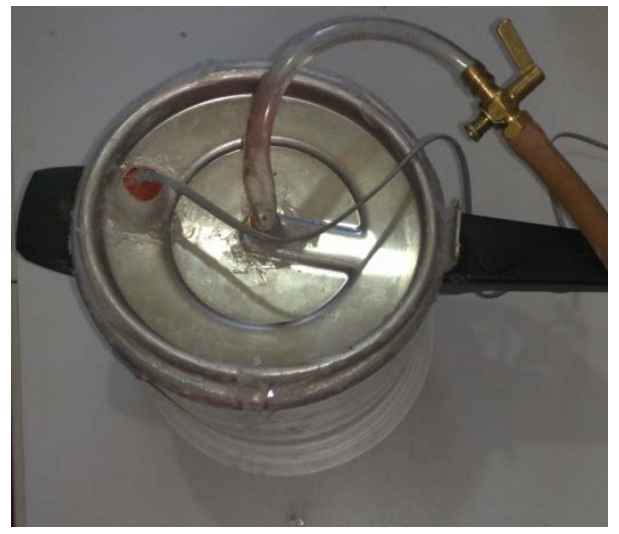

Figura 19 - Câmara de vácuo fechada
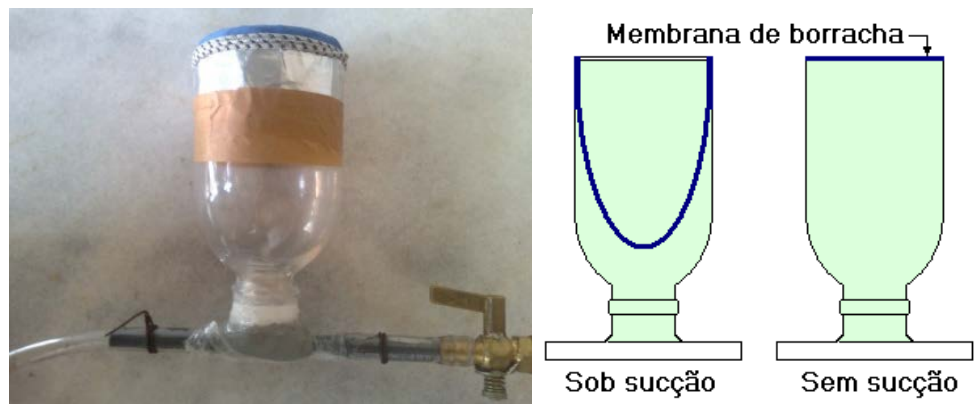

Figura 20 - Indicador de vácuo e válvula

16 Roriz, V. F.; Caram, R. M.; Roriz, M. 
A Figura 21 mostra o conjunto das temperaturas registradas em um dos experimentos.

A Figura 22 destaca detalhes do conjunto de dados, correspondentes aos momentos em que a temperatura da câmara é abruptamente alterada. Observa-se nesses detalhes que a amostra com fator de forma de 0,25 é a que segue mais de perto a alteração da temperatura da câmara, seguida pela amostra com fator de forma de 0,5 e pelas amostras com fator de forma 0,75 e 1, entre as quais praticamente não há diferença de temperaturas. Considerando que as amostras são idênticas quanto a sua composição e condições, a explicação para esse comportamento é o maior fluxo de calor por radiação entre a câmara e a amostra que tem o menor fator de forma (maior área exposta), enquanto o fluxo é menor para as amostras cujos fatores de forma são mais altos. No período de resfriamento, as temperaturas das amostras são mais altas que a da superfície da câmara e, portanto, o comportamento diferenciado entre as amostras indica diferenças entre suas emitâncias efetivas. Durante o aquecimento, esse processo inverte-se, passando a prevalecer as diferenças entre as absortâncias efetivas. Esses fatos permitem deduzir que os fatores de forma influenciam tanto as emitâncias quanto as absortâncias efetivas, o que já seria de se esperar, uma vez que aqueles efeitos resultam de acréscimos que as irregularidades superficiais provocam nas áreas de absorção e emissão de calor.

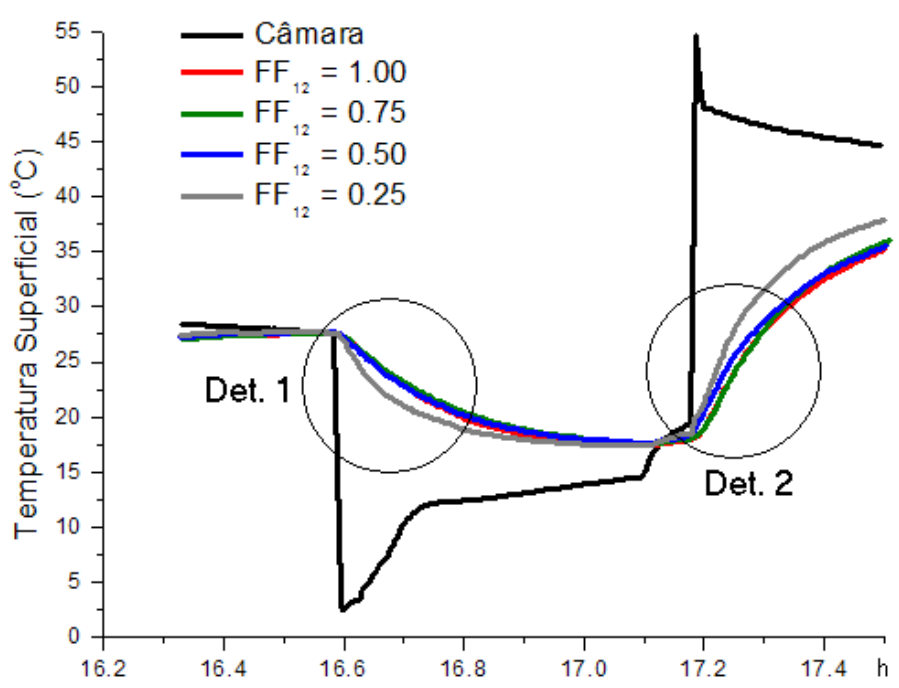

Figura 21 - Temperaturas registradas, destacando os momentos de resfriamento (Det. 1) e de aquecimento (Det. 2)
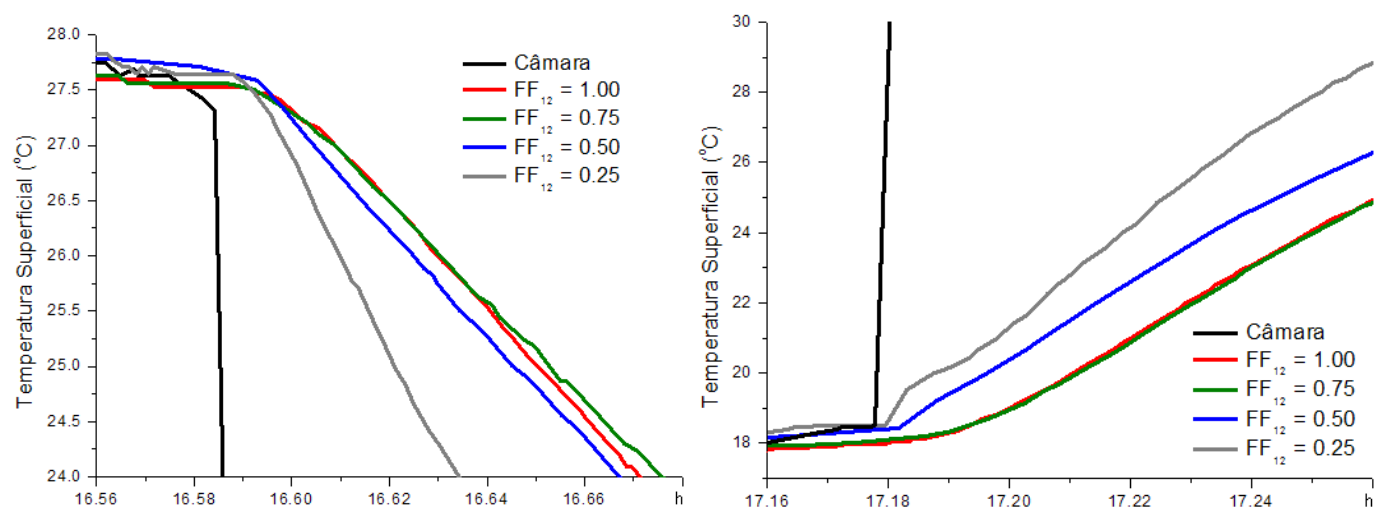

Figura 22 - Detalhes dos momentos de súbito resfriamento e aquecimento 


\section{Simulação computacional}

Para exemplificar a importância de tais aspectos, simulações foram elaboradas no programa EnergyPlus (UNITED..., 2012) para uma edificação de geometria simples, submetida ao clima da cidade de Brasília (DF). Simularam-se simultaneamente dois ambientes idênticos, exceto quanto às propriedades radiativas da cobertura (absortância e emitância). Em uma zona denominada Z1, adotou-se que as absortâncias são iguais às absortividades, e as emitâncias, iguais às emissividades, caso de superfície perfeitamente lisa e plana. Para uma segunda zona (Z2), consideraram-se as correções propostas pelas equações 15 e 19, e cobertura composta por telhas, conforme a Figura 23, de onde é possível obter o fator de forma $\left(\mathrm{FF}_{12}\right)$ pela Equação 21.

$$
F F_{12}=\left(\frac{A_{2}}{A_{1}}\right) \approx \frac{1964}{3002}=0.65
$$

Eq. 21

Para ambas as zonas, adotaram-se paredes de 10 cm de alvenaria cerâmica e piso de $5 \mathrm{~cm}$ de concreto.

\section{Primeiro exemplo}

Em um primeiro caso, considerou-se o edifício como um paralelepípedo de 6x9x2,8 m, sem janelas, portas ou aberturas envidraçadas (Figura 24). Conforme apresentado na norma NBR 15575 (ABNT, 2008), definiu-se um fluxo de ar de 1 renovação por hora e desconsideraram-se cargas térmicas internas. Considerando a emissividade da telha de 0,9, através da Equação 15 obtém-se uma emitância efetiva de 0,933. Da mesma forma, considerando uma absortividade de 0,6, pela Equação 19 obtém-se uma absortância efetiva de 0,698. A princípio, desconsiderou-se o fluxo com o solo, simulando os casos de dia típico de verão e inverno. A Tabela 2 apresenta as propriedades dos elementos construtivos adotadas nas simulações.

As temperaturas do ar internas às zonas simuladas Z1 (perfeitamente lisas e planas) e Z2 (admitindo as correções das equações 15 e 19) e as temperaturas do ar externo (TBS) são apresentadas na Figura 25.

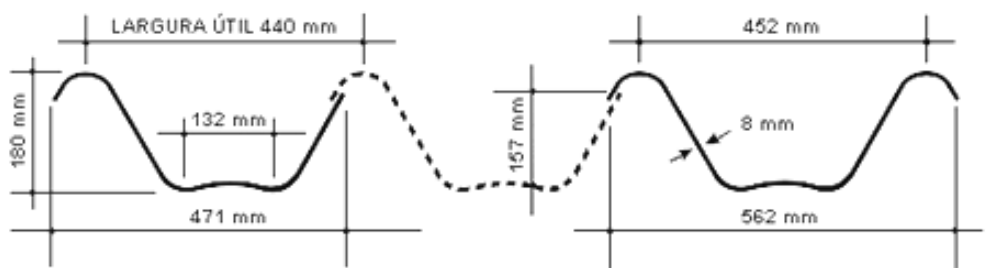

Figura 23 - Geometria da telha adotada nos exemplos

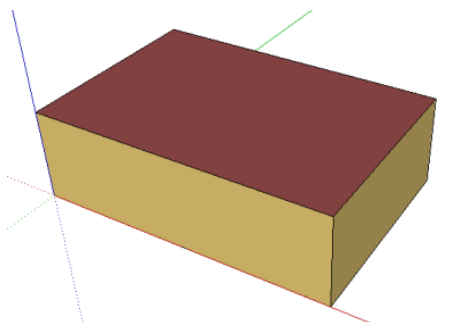

Figura 24 - Geometria do ambiente simulado nos casos dos exemplos 1 a 3

Tabela 2 - Características dos componentes utilizados nas simulações computacionais

\begin{tabular}{c|l|c|c|c|c|c|c}
\hline Exemplo & Componente & $\begin{array}{c}\text { Espessura } \\
\mathbf{( m )}\end{array}$ & $\begin{array}{c}\text { Condutividade } \\
\text { térmica } \\
\mathbf{( W / m . K )}\end{array}$ & $\begin{array}{c}\text { Densidade } \\
\mathbf{( k g / m 3 )}\end{array}$ & $\begin{array}{c}\text { Calor } \\
\text { específico } \\
\mathbf{( J / k g . K )}\end{array}$ & Emitância & Absortância \\
\hline & Paredes & 0,1 & 0,75 & 1.500 & 960 & 0,9 & 0,4 \\
& Piso & 0,05 & 1,7 & 2.300 & 960 & 0,9 & 0,4 \\
\hline $\mathbf{1}$ & Telha 1 & 0,006 & 1,7 & 2.300 & 960 & 0,9 & 0,6 \\
Fibrocimento & Telha 2 & 0,006 & 1,7 & 2.300 & 960 & 0,933 & 0,698 \\
\hline \multirow{2}{*}{ Fibrocimento } & Telha 3 & 0,006 & 1,7 & 2.300 & 960 & 0,9 & 0,3 \\
\hline 3 Metálica & Telha 4 & 0,006 & 1,7 & 2.300 & 960 & 0,933 & 0,397 \\
\hline & Telha 5 & 0,001 & 52 & 7.780 & 460 & 0,3 & 0,3 \\
\hline
\end{tabular}

18 Roriz, V. F.; Caram, R. M.; Roriz, M. 
É possível observar nesses resultados (Figura 25) a diferença nas temperaturas internas das zonas, as quais alcançam uma diferença máxima de $1,46{ }^{\circ} \mathrm{C}$, ocorrida nos momentos de temperatura máxima.

\section{Segundo exemplo}

Considerando uma cobertura pintada em cor clara, com absortividade de 0,3, pela Equação 18 obtémse uma absortância efetiva de 0,397 e, mantendose as demais características do exemplo 1, obtêmse resultados como os apresentados na Figura 26.

Observa-se, neste caso, uma diferença máxima de temperaturas de $1,62{ }^{\circ} \mathrm{C}$, nos mesmos momentos do exemplo anterior.

\section{Terceiro exemplo}

Considerando uma cobertura metálica clara, de mesma geometria que a anterior, com absortividade e emissividade de 0,3, obtêm-se absortância e emitância efetivas de 0,397. Considerando a zona Z1 coberta com a telha 5 e a zona Z2 coberta com a telha 6, descritas na Tabela 2, e mantendo-se as demais características do exemplo 1, as diferenças entre as temperaturas simuladas também são significativas no caso noturno, conforme apresentado na Figura 27.

As diferenças de temperaturas $\left(\mathrm{T}_{\mathrm{Z} 2}-\mathrm{T}_{\mathrm{Z} 1}\right)$ ficam entre $1,07{ }^{\circ} \mathrm{C}$ e $-0,78{ }^{\circ} \mathrm{C}$, sendo possível observar também divergências nas temperaturas mínimas desses ambientes.

Obteve-se, neste caso, uma diferença máxima de temperaturas $\left(\mathrm{T}_{\mathrm{Z} 2}-\mathrm{T}_{\mathrm{Z} 1}\right)$ de $2,83^{\circ} \mathrm{C}$.

\section{Quarto exemplo}

Neste caso, considerou-se o edifício como um paralelepípedo de $6 \times 9 \times 2,8 \mathrm{~m}$, com $4 \mathrm{~m} 2$ de janelas sombreadas voltadas para o norte, conforme a Figura 28. Manteve-se 1 renovação por hora e consideraram-se cargas internas de pessoas e equipamentos de uma residência, de maneira simplificada, conforme apresenta a Tabela 3.

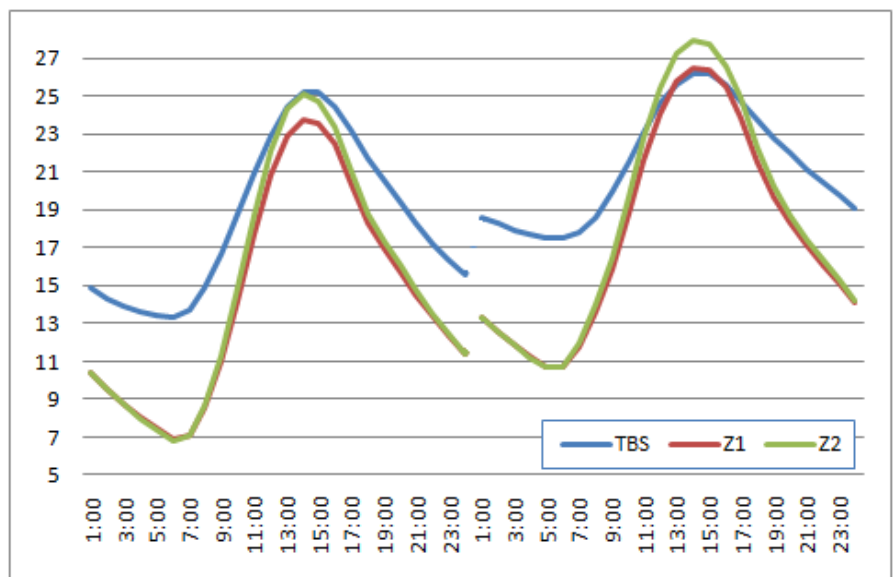

Figura 25 - Temperaturas obtidas na simulação do caso 1

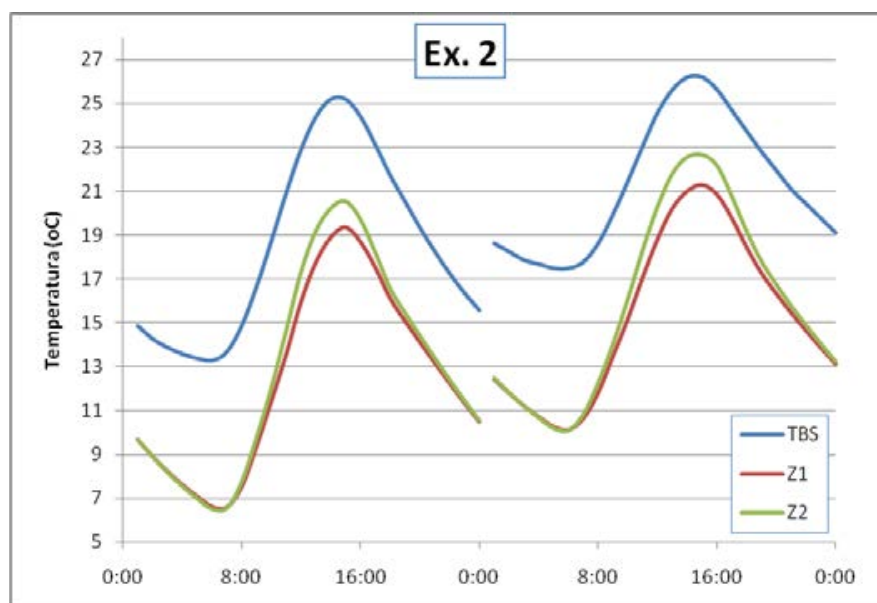

Figura 26 - Temperaturas obtidas na simulação do caso 2 


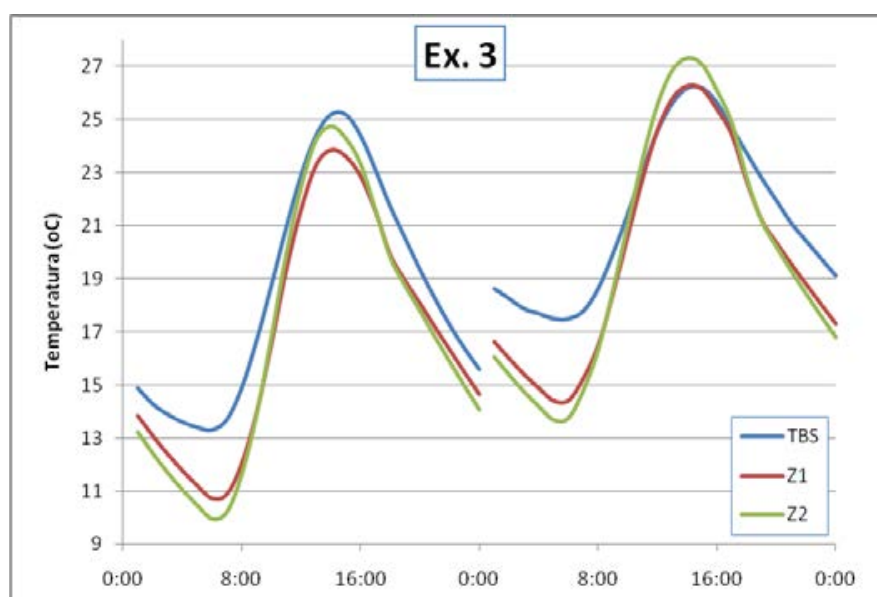

Figura 27 - Temperaturas obtidas na simulação do caso 3

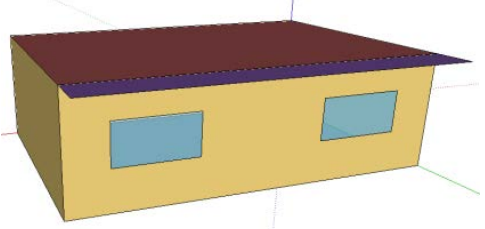

Figura 28 - Geometria do ambiente simulado no exemplo 4 (fachada norte)

Tabela 3 - Cargas térmicas consideradas

\begin{tabular}{l|l|l|l|l|l|l|l|l|l|l|l|l|l|l|l|l|l|l|l|l|l|l|l|l}
\hline \multicolumn{10}{c}{ Número de pessoas em atividade sedentária (x100 W) } \\
\hline Hora & 1 & 2 & 3 & 4 & 5 & 6 & 7 & 8 & 9 & 10 & 11 & 12 & 13 & 14 & 15 & 16 & 17 & 18 & 19 & 20 & 21 & 22 & 23 & 24 \\
\hline Sala & & & & & & & & & & & 1 & 2 & 1 & & 1 & 2 & 3 & 4 & 4 & 4 & 4 & 4 & 2 & 2 \\
\hline Cozinha & & & & & & 1 & 1 & 1 & 1 & 1 & 1 & 1 & 1 & 1 & 1 & 1 & 1 & & & & & & & \\
\hline Dormitório 1 & 2 & 2 & 2 & 2 & 2 & 1 & 1 & & & & & & & & & & & & & & & & 1 & 1 \\
\hline Dormitório 2 & 2 & 2 & 2 & 2 & 2 & 1 & 1 & & & & & & & & & & & & & & & & 1 & 1 \\
\hline
\end{tabular}

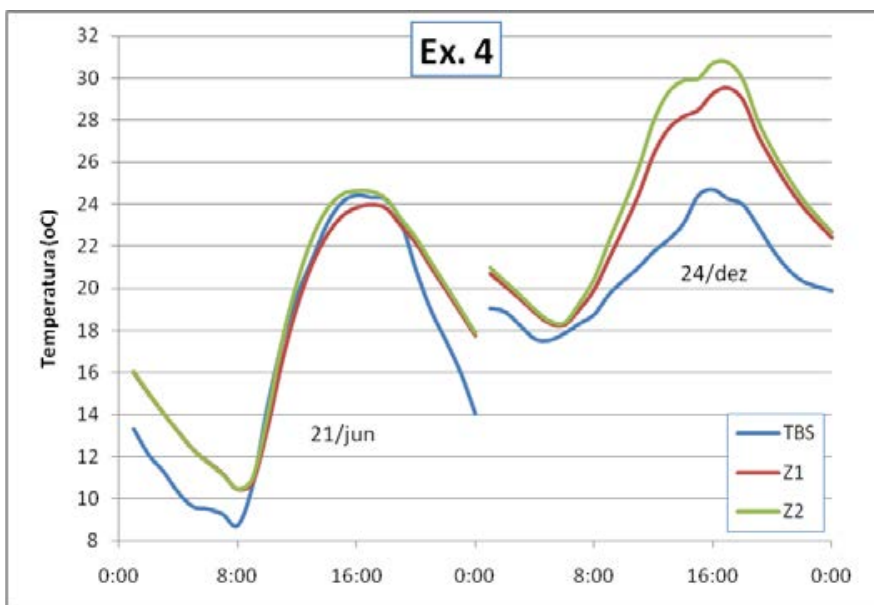

Figura 29 - Temperaturas obtidas na simulação do caso 4 


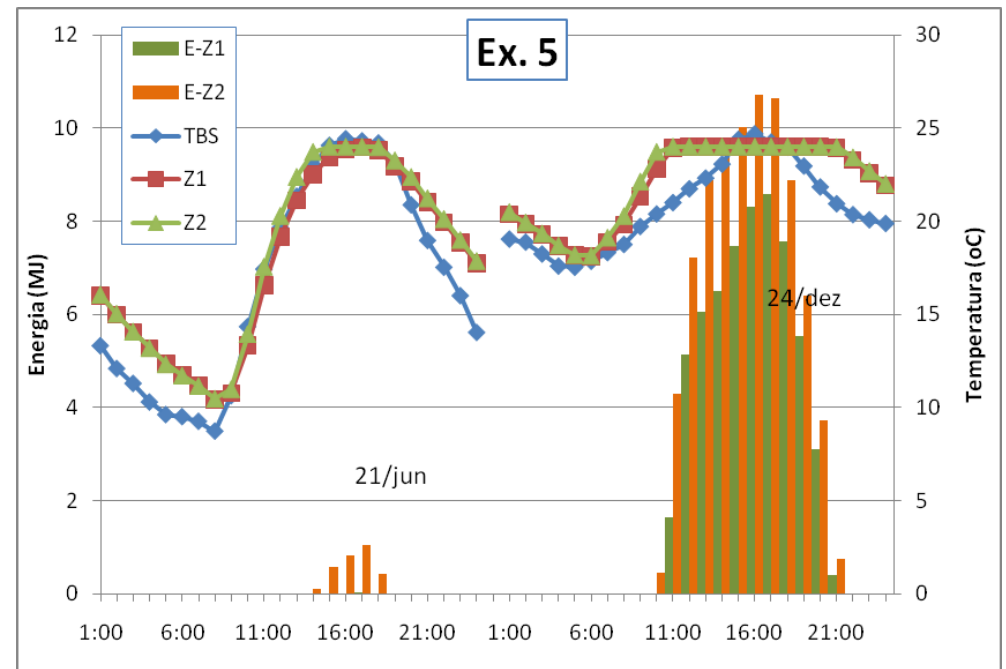

Figura 30 - Temperaturas obtidas na simulação do caso 5

Adotaram-se as telhas do exemplo 2, com absortividade de 0,3 e emissividade 0,9, e desconsiderou-se o fluxo com o solo.

Simulou-se o arquivo climático EPW de Brasília (UNITED..., 2012) para o ano todo. A Figura 29 ilustra os resultados obtidos.

Obteve-se, neste caso, uma diferença máxima de temperaturas $\left(\mathrm{T}_{\mathrm{Z} 2}-\mathrm{T}_{\mathrm{Z} 1}\right)$ de $2,83^{\circ} \mathrm{C}$.

\section{Quinto exemplo}

Para as condições do exemplo 4, simulou-se o uso de condicionadores de ar ideais em ambas as zonas, a fim de avaliar seus impactos no consumo de energia. Adotou-se limite de $24{ }^{\circ} \mathrm{C}$ para a temperatura interna máxima e comparou-se a soma dos consumos necessários para manter as temperaturas internas abaixo desse valor durante todo o ano. As temperaturas internas obtidas e respectivas cargas de refrigeração são ilustradas na Figura 30.

Para a zona Z1, a soma da carga anual ficou em 43.496 MJ, enquanto para a zona Z2 esse valor ficou em 56.401 MJ, apresentando. Assim. um acréscimo de 30\% em relação à verificada em Z1.

\section{Conclusões}

As variáveis em estudo apresentam alta relevância para avaliações do conforto térmico e da eficiência energética dos ambientes construídos.

Para as superfícies normalmente encontradas em edificações, o modelo simplificado aqui descrito apresenta boa correlação com o modelo clássico desenvolvido por Sparrow e Jonsson, tendo em alguns casos resultados idênticos. Apresenta, ainda, a vantagem de não ser restrito a apenas algumas formas.

Os resultados das simulações revelaram diferenças acima de 2,5 ${ }^{\circ} \mathrm{C}$ nas temperaturas internas do ar entre os cálculos que consideram ou não as correções propostas para as propriedades efetivas das superfícies. No caso do uso de condicionadores de ar, a diferença encontrada entre as estimativas de consumo de energia chegou a 30\%.

Para uso corrente dessas correções e real aprimoramento dos cálculos e estimativas termoenergéticas, a principal dificuldade está no desconhecimento das absortividades e emissividades das telhas, tintas e demais materiais em uso na construção.

\section{Referências bibliográficas}

\author{
ASSOCIAÇÃO BRASILEIRA DE NORMAS \\ TÉCNICAS. NBR 15575: edifícios habitacionais \\ de até cinco pavimentos: desempenho. Rio de \\ Janeiro, 2008.
}

\section{AMERICAN SOCCIETY FOR TESTING}

MATERIALS. C 1371: standard test method for determination of emittance of materials near room temperature using portable emissometers. West Conshohocken, 2004.

\section{AMERICAN SOCCIETY FOR TESTING MATERIALS. C 1549: standard test method for determination of solar reflectance near ambient temperature using a portable solar reflectometer. West Conshohocken, 2009.}

BERDAHL, P.; BRETZ, S. E. Preliminary Survey of the Solar Reflectance of Cool Roofing

Materials. Energy and Buildings, v. 25, n. 2, p. 49-158, 1997. 
JAPANESE STANDARDS ASSOCIATION: JIS A 1423: simplified test method for emissivity by infrared radio meter. Tokyo, 1983.

MAXIM. Datasheet. 2011. Disponível em:<http://www.maxim-

ic.com/datasheet/index.mvp/id/2812>. Acesso em: 12 mar. 2011.

RORIZ, V. F. Efeitos de Ondulação e Rugosidade de Superfícies Sobre Suas Absortâncias e Emitâncias: modelo teórico e experimental. Campinas, 2011. Tese (Doutorado em Engenharia Civil) - Faculdade de Engenharia Civil, Arquitetura e Urbanismo, Universidade Estadual de Campinas, Campinas, 2011.

SEKER, D. Z.; TAVIL, A. Ü. Evaluation of Exterior Building Surface Roughness Degrees by Photogrammetric Methods. Building and Environment, n. 31, n. 4, p. 393-398, jul. 1996.
SPARROW, E. M.; CESS, R. D. Radiation Heat Transfer. London: Hemisphere, 1978.

SPARROW, E.; JONSSON, V. Radiant Emission Characteristics of Diffuse Conical Cavities.

Journal of the Optical Society of America, n. 53, n. 7, p. 816-821, jul. 1963.

UNITED STATE. Department of Energy. Building Energy Software Tools Directory. Disponível em:

<http://www.eere.energy.gov/buildings/tools_direc tory/alpha_list.cfm>. Acesso em: 29 maio 2012.

\section{Agradecimentos}

Os autores agradecem à Fundação de Amparo à Pesquisa do Estado de São Paulo (Fapesp), pela bolsa de doutorado que permitiu o desenvolvimento da pesquisa.

Revista Ambiente Construído

Associação Nacional de Tecnologia do Ambiente Construído

Av. Osvaldo Aranha, 99 - 3o andar, Centro

Porto Alegre - RS - Brasil

CEP $90035-190$

Telefone: +55 (51) 3308-4084

Fax: +55 (51) 3308-4054

www. seer. ufrgs. br/ ambienteconstruido

E-mail: ambienteconstruido@ufrgs.br 